\title{
Communicating with Energy Harvesting Transmitters and Receivers
}

\author{
Kaya Tutuncuoglu Aylin Yener \\ Wireless Communications and Networking Laboratory (WCAN) \\ Electrical Engineering Department \\ The Pennsylvania State University, University Park, PA 16802 \\ kaya@psu.edu_yener@ee.psu.edu
}

\begin{abstract}
This paper provides a general framework for utility maximization of a wireless network with energy harvesting nodes. The focus is on applying this framework to the single-link problem with an energy harvesting transmitter and an energy harvesting receiver. For the general utility maximization problem, it is shown that if the utility of a network can be expressed instantaneously as a function of the powers of the nodes, then the maximum utility achieving power policy for each node can be found using a water-filling approach for each user. This is achieved by expressing the general utility maximization problem as a pair of nested problems focusing on energy efficiency and adapting to energy harvests separately. The framework extends the previous results on offline optimization of energy harvesting transmitters to networks with all energy harvesting nodes including receivers and relays as well as any network utility, provided that the achieved utility is instantaneous and additive in time. The implications of the energy efficiency problem on the energy harvesting problem are demonstrated over an energy harvesting transmitter-receiver pair, and simulation results are presented to exhibit the performance of the optimal policy along with some alternatives for a range of storage capacities.

Index Terms-Energy harvesting, utility maximization, wire-
\end{abstract} less networks, optimal scheduling, battery limited nodes.

\section{INTRODUCTION}

With the recent advances in energy harvesting devices and an increasing demand for environment friendly mobile communications, energy harvesting is emerging as a desirable alternative as a means for wireless networks to function. Whether the objective is to decrease the carbon footprint of wireless communications or to make nodes energy-wise selfsufficient, harvesting energy from external sources is promising. On the other hand, the availability of ambient energy is time-varying and limited in most applications. Therefore, utilizing this energy efficiently becomes an important problem to match the performances of their battery or grid powered counterparts. Recently, there has been a substantial research effort on optimal scheduling for energy harvesting transmitters in single and multiple user settings [1]-[12]. In this paper, we present a generalized solution that applies to many scenarios and problems considered in the previous work, and beyond.

An offline scheduling problem with a discrete energy harvesting model was first introduced in [1], solving the transmission completion time minimization problem for a transmitter with infinite energy storage. This work was later extended in [2] to transmitters with limited energy storage, also showing the duality between transmission completion time minimization and short-term throughput maximization problems in the energy harvesting setting. A directional waterfilling algorithm that also considers channel variations was developed in [3], focusing on a Gaussian fading channel with an energy harvesting transmitter. This approach was subsequently extended to other network models including the broadcast channel [4], [5], multiple access channel [6], and interference channel [7]. A common theme in these papers was the achieved instantaneous rate being a function of the instantaneous transmission power of the nodes.

More involved models also emerged for energy harvesting networks in the offline setting, such as the two-hop model with energy harvesting transmitter and relay [8], and models with inefficient energy storage [9], [10]. Parallel to these efforts, online approaches with causal energy and state information were also considered with a queueing theoretic approach in [11], and a Markovian energy model approach in [12].

The aforementioned previous work has focused on an energy harvesting transmitter, ignoring other computational or operational power costs on both sides of a channel. It is argued in [13] that receiver decoding power can be significant in specific applications, and that there is a fundamental trade-off between transmitter and decoding power. Arguably receiver power consumption is even more critical in the energy harvesting setting.

In this paper, we provide a general solution to the collection of offline optimization problems maximizing the integral of any instantaneous utility, for any network model such as in [1]-[7]. The formalization presented enables handling possible transmitter-receiver power tradeoffs, as well as transmitter and receiver side practicalities including storage inefficiency and practical transmission schemes, e.g., adaptive modulation techniques [14], [15]. This is achieved by decoupling the overall optimization problem to two nested problems focusing on two different aspects of the problem, energy efficiency and adapting to energy harvests. The energy harvesting and network models and the general solution is presented in Section II and Section III respectively. The special case with an energy harvesting transmitter and an energy harvesting receiver is considered in Section IV. Numerical results to demonstrate 


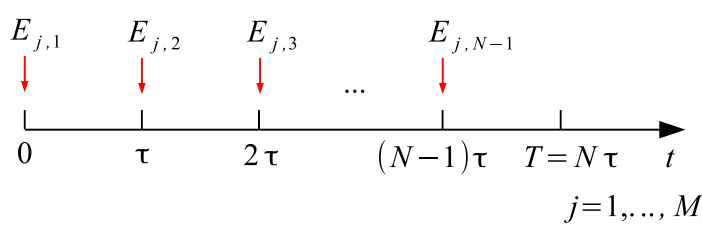

Fig. 1: Energy harvesting model for the $j^{t h}$ network node.

the performance for the energy harvesting transmitter-energy harvesting receiver pair are presented Section V. The paper is concluded in Section VI.

\section{SySTEM MODEL}

We consider $M$ energy harvesting network nodes, each harvesting and storing their own energy throughout the operation of the network. These nodes can be sensors, transmitters, relays and receivers, each operating within their own energy budget to improve the utility of the network. For simplicity, we assume a time slotted model of slot duration $\tau$, where the channel can be assumed constant throughout each time slot. The state of the channel is represented by the vector $\boldsymbol{\alpha}_{i}$ in time slot $i$. This vector can, for instance, correspond to the fading state, in which case our assumption would require that the coherence time of the channel is longer than a time slot. We study a system with an information delivery deadline $T=N \tau$.

As the setting suggests, the energy harvesting process is varying and different for each user. The $j^{\text {th }}$ energy harvesting node harvests an energy packet of $E_{j, i} \geq 0$ at the beginning of the $i^{t h}$ time slot, i.e., at time $i \tau$, available immediately for consumption. The arrival scenario is depicted in Figure 2. Each network element is assumed to have a battery or high energy density capacitor of capacity $E_{j}^{\max }$ for storage. Any arrival exceeding this capacity is truncated at $E_{j}^{\max }$ for simplicity, since the overflowing energy is certainly lost.

The offline scheduling problem is considered in this paper, indicating that the energy arrivals $E_{j, i}$ and the channel parameters $\boldsymbol{\alpha}_{i}$ are assumed to be known prior to transmission. The purpose of this assumption is to quantify the benchmark performance, provide insights in development of online policies, and apply to practical cases where these parameters can be estimated in an accurate manner.

Each energy harvesting node utilizes its harvested energy towards the network objective, aiming to improve the overall utility of the network. The rate of energy consumption at each node is limited by its harvesting process and storage capacity. Denoting the instantaneous power consumption of node $j$ with the non-negative integrable function $p_{j}(t)$, the cumulative energy consumed by node $j$ at any time $t$ falling in the $i^{t h}$ time slot is upper bounded by the harvested energy as

$$
\int_{0}^{t} p_{j}(t) d t \leq \sum_{k=0}^{i} E_{j, k}
$$

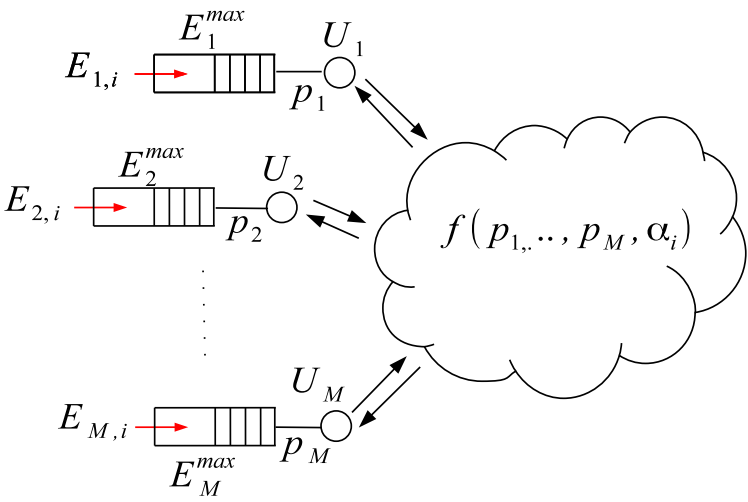

Fig. 2: The generalized network model with $\mathrm{M}$ nodes and utility $f\left(\mathbf{p}, \boldsymbol{\alpha}_{i}\right)$ at time slot $i$.

and lower bounded due to the battery capacity as

$$
\sum_{k=0}^{i} E_{j, k}-\int_{0}^{t} p_{j}(t) d t \leq E_{j}^{\max }
$$

for all time instants $0 \leq t \leq T$ and users $j=1, \ldots, M$. The constraint in (2) follows from the observation that allowing any arriving energy to overflow is undesirable. The unlikely case where discarding energy at a node is better for the network will be handled alternatively in the sequel by manipulating the utility function instead.

We wish to formulate the problem in a general fashion in order to cover a larger set of optimization problems. In previous work [1]-[7], the utility to be optimized has been the reliable communication rate, or the closely related transmission completion time. The achievable rates and capacity for most channels considered in these papers are well-known, allowing customized solutions to the problem. However the capacity of an arbitrary network model with $M$ users is an open problem. We define a general utility function,

$$
f(\mathbf{p}, \boldsymbol{\alpha})=f\left(p_{1}, p_{2}, \ldots, p_{M}, \boldsymbol{\alpha}\right)
$$

which yields the instantaneous utility of the network given the instantaneous powers of nodes as $p_{j}$ and the related channel parameter as the vector $\boldsymbol{\alpha}$. This utility can be replaced with any instantaneous and additive performance metric depending on the application, some examples of which are instantaneous rate, average distortion, or successful transmission probability. There is no restriction on the utility function in (3) other than being integrable, since the problem will aim to maximize the integral of this function. Hence the model is applicable to a wide range of scenarios, due to the freedom of choice in both the network topology and objective function.

With the instantaneous utility and energy constraints defined above, the average utility maximization problem is expressed as

$$
\begin{array}{rlr}
\max _{\mathbf{p}(t) \geq 0} & \frac{1}{T} \int_{0}^{T} f\left(\mathbf{p}(t), \boldsymbol{\alpha}_{i}\right) d t, & i=\left\lfloor\frac{t}{\tau}\right\rfloor \\
\text { s.t. } & p_{j}(t) \in \mathfrak{P}_{j}, & j=1, \ldots, M
\end{array}
$$


where $i$ represents the time slot in which $t$ falls, and $\mathfrak{P}_{j}$ is the set of energy-feasible power policies $p_{j}(t)$ for the $j^{t h}$ user,

$$
\mathfrak{P}_{j}=\left\{p(t) \geq 0 \mid 0 \leq \sum_{k=0}^{i} E_{j, k}-\int_{0}^{i \tau} p_{j}(t) d t \leq E_{j}^{\max }, i \leq N\right\}
$$

Note that verifying the energy feasibility constraints at the beginning and end of time slots is sufficient. This is due to consumed powers $p_{i}(t)$ being non-negative by definition, causing the stored energy to reach its local extremum at the beginning and end of the time-slots. Since harvested energy remains constant within a time slot, feasibility at $i \tau^{+}$and $(i+1) \tau^{-}$implies feasibility throughout time slot $i$. We next present a general solution to the utility maximization problem in (4).

\section{Decoupled Problem And AnAlysis}

In this section, we show that the problem defined in (4) can be decoupled into two nested problems that can be solved independently, with the two resulting problems separately targeting the aspects of energy efficient communication and adapting to the energy harvests. We begin by defining the energy consumed by node $j$ in time slot $i$ as

$$
\epsilon_{j, i}=\int_{i \tau}^{(i+1) \tau} p_{j, i}(t) d t
$$

and the collection of $\epsilon_{j, i}$ 's for all nodes at time slot $i$ as the vector $\epsilon_{i}$. This variable can be added to the parameters of the maximization in (4) with the definition in (6) as an equality constraint without altering the optimal solution, yielding the expanded problem

$$
\begin{aligned}
\max _{\mathbf{p}(t) \geq 0, \boldsymbol{\epsilon}_{i}} & \frac{1}{T} \int_{0}^{T} f\left(\mathbf{p}(t), \boldsymbol{\alpha}_{i}\right) d t, \quad i=\left\lfloor\frac{t}{\tau}\right\rfloor \\
\text { s.t. } & \epsilon_{j, i}=\int_{i \tau}^{(i+1) \tau} p_{j, i}(t) d t \\
& p_{j}(t) \in \mathfrak{P}_{j}, \quad j=1, \ldots, M .
\end{aligned}
$$

We present the following observation in order to decouple the maximization over the two set of variables.

Lemma 1: The optimal total consumed energy values $\boldsymbol{\epsilon}_{i}$ in the $i^{\text {th }}$ time slot is sufficient to determine the power policy within the time slot in consideration, i.e., $\mathbf{p}(t)$ for $t \in[i \tau,(i+$ 1) $\tau$ ]. The optimal policy within this time slot can be found as the solution to the total energy constraint problem,

$$
\begin{aligned}
\max _{\mathbf{p}(t)} & \int_{i \tau}^{(i+1) \tau} f\left(\mathbf{p}(t), \boldsymbol{\alpha}_{i}\right) d t, \\
\text { s.t. } & \epsilon_{j, i} \geq \int_{i \tau}^{(i+1) \tau} p_{j, i}(t) d t, \quad i=1, \ldots, M,
\end{aligned}
$$

which will be referred to as the energy efficiency problem or the inner problem in the sequel.

The proof of above lemma follows from the fact that unless the power policy maximizes the achieved utility within the said time slot for a given total energy $\epsilon_{i}$, it can be replaced with a better one within $[i \tau,(i+1) \tau]$ without violating the energy constraints of the original problem given in (5).

We denote by $U(\boldsymbol{\epsilon}, \boldsymbol{\alpha})$ the result of the maximization in (8), i.e., the maximum utility that can be achieved in a time slot with total energy constraint vector $\epsilon$ and channel parameter $\boldsymbol{\alpha}$. Since we know that this will be the achieved utility for the optimal policy and that it satisfies the constraints in (7b), we can restate the problem in (7) as

$$
\begin{aligned}
& \max _{\boldsymbol{\epsilon}_{i} \geq 0} \frac{1}{T} \sum_{i=1}^{N} U\left(\boldsymbol{\epsilon}_{i}, \boldsymbol{\alpha}_{i}\right), \\
& \text { s.t. } \quad 0 \leq \sum_{k=0}^{i} E_{j, k}-\epsilon_{j, k} \leq E_{j}^{\max }, \\
& \qquad i=0, \ldots, N-1 .
\end{aligned}
$$

We will refer to this problem as the energy allocation problem or the outer problem on account of being dependent on the values of $U(\boldsymbol{\epsilon}, \boldsymbol{\alpha})$. Note that the energy feasibility constraints on the consumed power vector in (7c) are now replaced with an equivalent set of constraints on $\epsilon_{i}$ in (9b). This is possible due to $\epsilon_{i}$ being sufficient to determine the available energy values at $i \tau$ for any $i$.

In order to gain insight on how the energy allocation problem in (9) can be solved, we focus on characterizing its objective function. We start by pointing out that $U(\boldsymbol{\epsilon}, \boldsymbol{\alpha})$ is concave in $\boldsymbol{\epsilon}$ for a fixed $\boldsymbol{\alpha}$. This is a direct consequence of (8); any point violating concavity of $U$ can be improved by time-sharing, concavifying the function without violating the total energy constraint since the constraint is linear. Another property is that $U(\boldsymbol{\epsilon}, \boldsymbol{\alpha})$ is non-decreasing in $\boldsymbol{\epsilon}$, which follows from the inequality in (8b), indicating that an energy vector $\epsilon_{1}$ must perform at least as good as any $\epsilon<\epsilon_{1}$. This means that if any energy is to be discarded in the optimal policy, this will be handled by the inner problem by choosing a policy that is not on the boundary of (8b), justifying the storage capacity constraint in (2).

Together with (9b) yielding a convex constraint set, it can be stated that the energy allocation problem is convex, and the solution satisfies the KKT conditions. As found for special cases in the previous work [4], [6], [7], the solution to this problem in each dimension can be obtained by generalized directional water-filling algorithm, with water-levels varying throughout the transmission period with

$$
v_{j, i}=\left.\frac{\partial}{\partial \epsilon_{j}} U\left(\boldsymbol{\epsilon}, \boldsymbol{\alpha}_{i}\right)\right|_{\epsilon_{j, i}}=\sum_{k=i}^{N}\left(\lambda_{j}-\mu_{j}\right)-\eta_{i}
$$

where $\lambda, \mu$, and $\eta$ are the non-negative Lagrangian multipliers for the energy constraints, taking positive values when the stored energy is 0 or $E_{j}^{\max }$, and the non-negativity constraint on $\epsilon_{i}$ respectively. The water-levels are found by a constrained water-flow, the details of which can be found in [3] for a single link Gaussian fading channel. This is extended to the multinode case by alternating the maximization between the energy allocation of each node, which is known to converge due to the convexity of the problem, as done in [7]. 


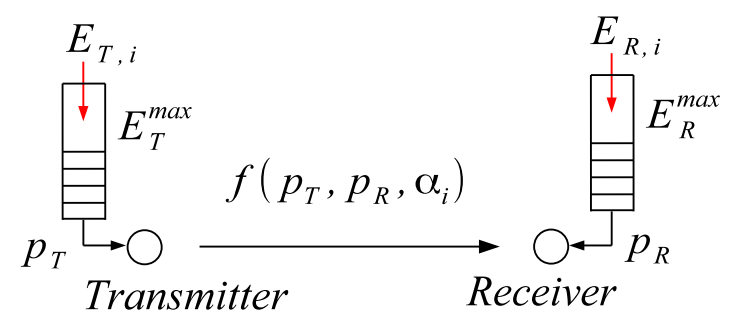

Fig. 3: A single link energy harvesting transmitter and receiver model.

\section{EXAmple: ENERgy HaRVESTING TRANSmitTer AND RECEIVER}

An immediate application of the framework described is a two-node network with an energy harvesting transmitter and energy harvesting receiver, shown in Figure 3. This case has not been studied to date, primarily due to the lack of general analytical models on receiver power consumption. Owing to the ability to handle arbitrary utility functions, our approach in Section III extends to this setting.

Consider the average communication rate as the utility of this system. Unlike the previous single-link work with ratebased utility such as in [2], [3] where achieved rate depends on the number of bits departed by the transmitter, we also need to consider how many bits the receiver can decode, process and possibly store within its own energy budget. Although the rate dependent power consumption of a receiver is not explicitly studied, recent work on decoding energy has shown that there is a trade-off between transmitter and receiver power when achieving a predetermined probability of error [13]. This is due to better channel coding requiring more energy for decoding, and simpler channel coding requiring more bits to transmit the same amount of information. Therefore the rate achieved can be modeled as a function of both transmitter and receiver powers reflecting this tradeoff. Furthermore, transmitters and receivers might have additional concerns that affect the utility, or in our case the rate of the system; such as power consumed for sensing, pre-processing and compressing the data on the transmitter side, and decompressing, processing, and storage on the receiver side. These factors together yield the utility or rate that can be achieved with a certain set of powers at the nodes, denoted by the utility function $f\left(p_{T}, p_{R}, \boldsymbol{\alpha}\right)$ in Figure 3.

For demonstration purposes, we assume a wireless sensor transmitter and a data center as receiver in a static AWGN channel. Consider the following power requirements by the nodes for successful operation: For device operation, a constant power of $p_{T}^{o n}$ and $p_{R}^{o n}$ is needed by the transmitter and receiver respectively to transmit or receive at any nonzero rate. Otherwise the node is assumed to be in a sleep state with negligible power consumption producing zero utility. To successfully depart bits at rate $r_{t}$, the transmitter requires a transmission power $P_{x}$ satisfying $r_{t}=B \cdot \log \left(1+k P_{x}\right)$ where

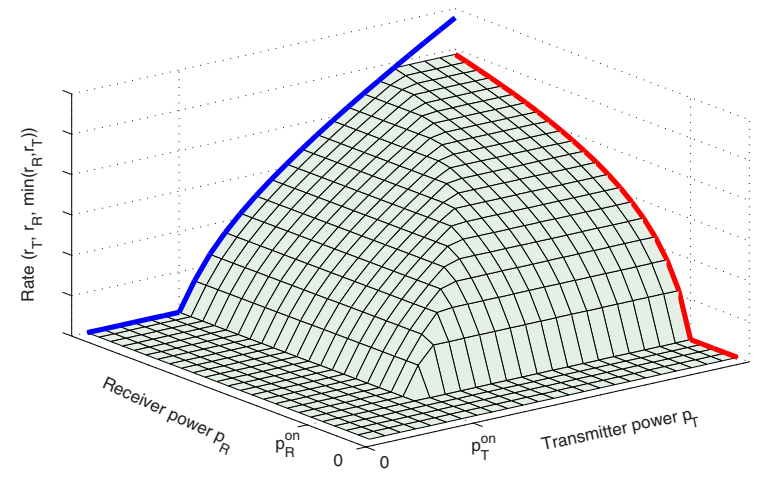

(a) Instantaneous rates for transmitter, receiver and system

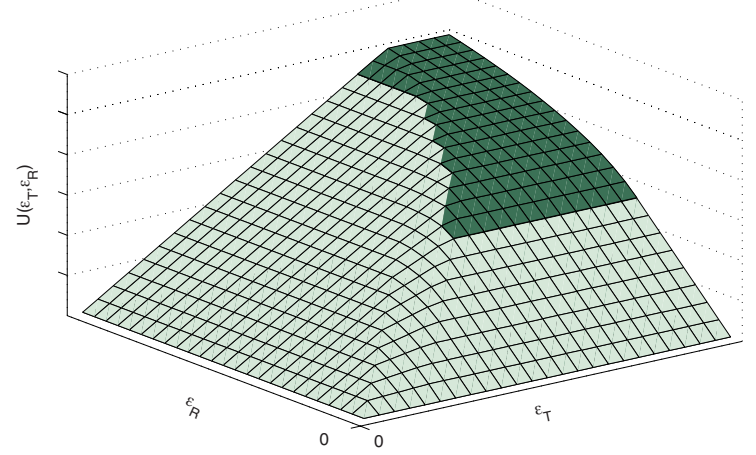

(b) Resulting solution to the inner problem, $U(\boldsymbol{\epsilon})$

Fig. 4: Plots of the utility function $r\left(p_{T}, p_{R}\right)$ and the solution to the inner problem $U\left(\epsilon_{T}, \epsilon_{R}\right)$.

$k$ depends on received noise at the receiver and the path loss of the channel. On the receiver side, let the receiver require a decoding power exponential in the desired rate, and a circuit power linear in the rate of processing and storage in addition to $p_{R}^{o n}$. The resulting transmitter power-rate function $r_{T}\left(p_{T}\right)$ and receiver power-rate function $r_{R}\left(p_{R}\right)$ with some arbitrary parameters is shown in Figure 4a in blue and red respectively, along with the achieved rate-based utility $r\left(p_{1}, p_{2}\right)$ given as the minimum of the two achieved rates for a given power vector $\left(p_{T}, p_{R}\right)$.

Next, we solve the inner problem for the utility given in Figure $4 \mathrm{a}$ for a time slot of unit length. The inner problem essentially requires finding a possibly infinite set of points on $r\left(p_{1}, p_{2}\right)$ and corresponding weights that, when linearly combined, consume no more energy than the given energy pair $\left(\epsilon_{T}, \epsilon_{R}\right)$ while achieving the maximum rate on average. Conversely, this requires any point on $U\left(\epsilon_{T}, \epsilon_{R}\right)$ to be achievable by a linear combination while the overall function is concave. Thus, if we plot the naively achievable utilities as the bits communicated with constant power transmissions, $\tau \cdot r\left(\epsilon_{1} / \tau, \epsilon_{2} / \tau\right)$, the solution is the convex hull of this function as seen in Figure $4 \mathrm{~b}$. The darker region in this plot represents the values of $U\left(\epsilon_{T}, \epsilon_{R}\right)$ for which constant power transmission 
is sufficient to solve the inner problem, while the lighter region denotes utilities achieved by time-sharing. In effect, this means that when the allocated energy in a slot falls on the light colored region, the nodes are required to transmit with a power corresponding to a higher rate for some fraction of the time slot, and sleep in the remaining fraction to achieve the energy efficient utility.

With the optimal in-slot behavior of transmitter and receiver nodes described by the maximum utility function $U\left(\epsilon_{T}, \epsilon_{R}\right)$, what remains is to solve the outer problem using generalized directional water-filling alternatingly for the two nodes. This part of the problem allows the network to adopt to energy harvests by optimally allocating energy to slots, yielding the average rate maximizing power allocation policy. The solution requires alternating between the transmitter and receiver on finding the water levels $\mathbf{v}_{T}$ and $\mathbf{v}_{R}$ for both users given as,

$$
\begin{aligned}
& v_{T, i}=\left.\frac{\partial}{\partial \epsilon_{T}} U\left(\epsilon_{T}, \epsilon_{R}\right)\right|_{\epsilon_{T, i}}=\sum_{k=i}^{N}\left(\lambda_{T, k}-\mu_{T, k}\right)-\eta_{T, i} \\
& v_{R, i}=\left.\frac{\partial}{\partial \epsilon_{R}} U\left(\epsilon_{T}, \epsilon_{R}\right)\right|_{\epsilon_{R, i}}=\sum_{k=i}^{N}\left(\lambda_{R, k}-\mu_{R, k}\right)-\eta_{R, i}
\end{aligned}
$$

where $U\left(\epsilon_{T}, \epsilon_{R}\right)$ is the concavified function in Figure $4 \mathrm{~b}$ and $\lambda, \mu$ and $\eta$ are non-negative Lagrangian multipliers for each node, satisfying the complementary slackness conditions

$$
\begin{aligned}
& \lambda_{j, i}\left(\sum_{k=0}^{i} E_{j, k}-\epsilon_{j, k}\right)=0, \quad \eta_{j, i} \cdot \epsilon_{j, i}=0, \\
& \mu_{j, i}\left(\sum_{k=0}^{i} E_{j, k}-\epsilon_{j, k}-E_{j}^{\max }\right)=0 \quad j \in\{T, R\} .
\end{aligned}
$$

The two water-filling algorithms interact through $U\left(\epsilon_{T}, \epsilon_{R}\right)$, since the partial derivative of this function for one node changes with the allocated energy of the other node. The alternating algorithm converges to yield the optimal policy, for which the water levels for both nodes satisfy equations (11) and (12) simultaneously.

\section{Simulations}

In this section, we provide simulation results to account for the performance of the optimal and some alternative suboptimal transmitter and receiver policies. We simulate an energy harvesting transmitter receiver pair in a static AWGN channel for 1000 slots and a slot length of $\tau=1 \mathrm{sec}$. Both the transmitter and the receiver require a constant operating power of $p_{T}^{o n}=p_{R}^{o n}=50 \mathrm{~mW}$ for being awake. Additionally, the transmitter performs adaptive modulation for power control [14], [15] with a rectangular M-QAM constellation, achieving a discrete set of rates; while the receiver is required to process and store the received data, modeled with a constant energy cost of $5 \mathrm{~mJ} /$ bit, yielding the following individual rates achieved:

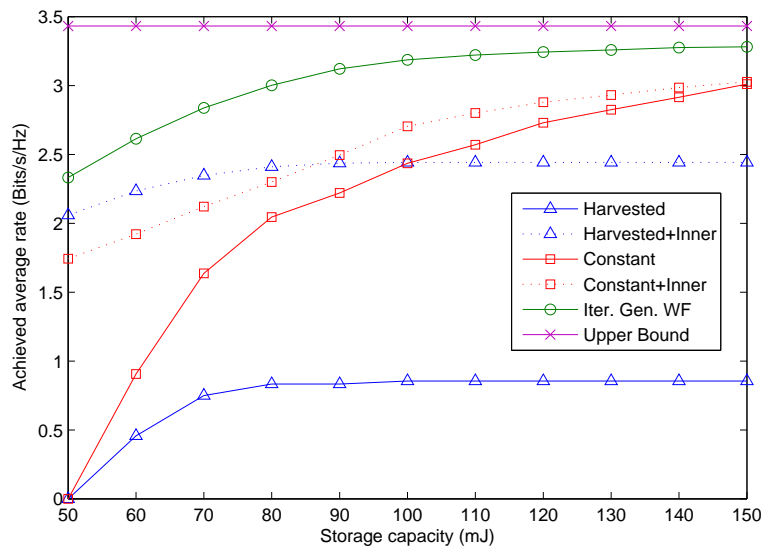

Fig. 5: Average rates achieved using optimal and proposed suboptimal policies for varying energy storage capacity.

$$
\begin{aligned}
& r_{T}(p)=\left\{\begin{array}{lll}
0, & {\left[p-p_{T}^{o n}\right]^{+}<1 m W(O F F)} \\
1, & p-p_{T}^{o n} \in[1,2) m W(B P S K) & \\
2, & p-p_{T}^{o n} \in[2,6) m W(Q A M) & \\
3, & p-p_{T}^{o n} \in[6,10) m W(8-Q A M) & \\
\vdots & \vdots
\end{array}\right. \\
& r_{R}(p)=\left[\frac{p-50 m W}{5}\right]^{+} \text {bits/s/Hz. }
\end{aligned}
$$

We define the system utility as $\min \left(r_{T}, r_{R}\right)$ when the two nodes achieve rates $r_{T}$ and $r_{R}$ simultaneously. In other words, the utility of this system is the average rate of successfully transmitted, processed and stored bits. The energy harvesting process is assumed to be an i.i.d. process, with $E_{T, i}$ and $E_{R, i}$ distributed uniformly in $[0,100] m J$, yielding an average harvesting rate of $50 \mathrm{~mW}$ per node.

The system is simulated with various power allocation policies, and the average utility achieved against the storage capacity of the nodes $\left(E_{T}^{\max }=E_{R}^{\max }\right)$ is plotted in Figure 5 . The blue lines correspond to algorithms that consume the harvested energy as it is received, labeled Harvested. The red lines represent a constant power algorithm that waits for an energy threshold and transmits with the optimal constant power whenever threshold is reached, indicated with Constant. Additionally, the two dashed plots labeled with an additional $T S$ term are allowed to use the time-sharing rates, i.e., can achieve the utility suggested by the output of the Inner problem. The performance of the nested optimization in this problem is shown by the green plot, with label Iter. Gen. $W F$ representing iterative generalized water-filling used in the outer problem. Finally, an upper-bound on average rate derived using a non-energy harvesting model with an average power of $50 \mathrm{~mW}$ per node is shown in magenta.

As expected, policies without optimal power allocation in each time-slot, i.e., without utilization of the time-sharing results of the energy efficiency problem, perform particularly 
worse for smaller storage capacities, since they rarely accumulate enough energy to just turn their circuitry on for an entire time-slot. When these algorithms start employing optimal time-sharing within the slots, shown in dashed lines, the performance is significantly increased. The optimal algorithm, using both time-sharing and optimal energy allocation with iterative water-filling, performs notably better than all alternatives, allowing the system to yield a utility close to the upperbound, especially when the storage is limited. With increasing storage capacity, the constant power transmitter is expected to further approach the proposed optimal policy; however the proposed policy allows using much smaller storage for the same performance.

\section{CONCLUSION}

This paper analyzes an energy harvesting transmitter energy harvesting receiver pair, and provides a method of developing optimal power policies by demonstrating that the optimization problem is an instance of a utility maximization framework for energy harvesting networks whose solution is provided. In particular, we provide a framework for optimizing energy harvesting networks with packet energy arrivals by decoupling the problem into two nested problems: an inner problem addressing energy efficiency and an outer problem addressing energy allocation for energy harvesting nodes. This approach yields a solution that can be extended to any network topology with an instantaneous, i.e., memoryless, utility function. For the application of this framework on the transmitter-receiver pair where both nodes are energy harvesting, numerical results reveal that the optimal policy performs notably closer to the upper bound, which is the utility of the non-energy harvesting system, i.e., when the nodes have the total energy available to them at the commencement of the session.

\section{REFERENCES}

[1] J. Yang and S. Ulukus, "Optimal packet scheduling in an energy harvesting communication system," IEEE Transactions on Communications, submitted Jun. 2010, available at http://arxiv.org/abs/1010.1295.
[2] K. Tutuncuoglu and A. Yener, "Optimum transmission policies for battery limited energy harvesting nodes," IEEE Transactions on Wireless Communications, submitted Sept. 2010, available at http://arxiv.org/abs/1010.6280.

[3] O. Ozel, K. Tutuncuoglu, J. Yang, S. Ulukus, and A. Yener, "Transmission with energy harvesting nodes in fading wireless channels: Optimal policies," IEEE Journal on Selected Areas in Communications, vol. 29, no. 8, pp. $1732-1743$, Sep. 2011.

[4] J. Yang, O. Ozel, and S. Ulukus, "Broadcasting with an energy harvesting rechargeable transmitter," IEEE Transactions on Wireless Communications, submitted Oct. 2010, available at http://arxiv.org/abs/1010.2993.

[5] O. Ozel, J. Yang, and S. Ulukus, "Optimal scheduling over fading broadcast channels with an energy harvesting transmitter," in Proceedings of the The Fourth International Workshop on Computational Advances in Multi-Sensor Adaptive Processing (CAMSAP), San Juan, Puerto Rico, Dec. 2011.

[6] J. Yang and S. Ulukus, "Optimal packet scheduling in a multiple access channel with rechargeable nodes," in Proceedings of the IEEE International Conference on Communications, ICC'11, Kyoto, Japan, Jun. 2011.

[7] K. Tutuncuoglu and A. Yener, "Sum-rate optimal power policies for energy harvesting transmitters in an interference channel," to appear in JCN Special Issue on Energy Harvesting in Wireless Networks, submitted October 2011, available at http://arxiv.org/abs/1110:6161.

[8] D. Gunduz and B. Devillers, "Two-hop communication with energy harvesting," to appear in Proceedings of the 4th International Workshop on Computational Advances in Multi-Sensor Adaptive Processing, (CAMSAP), Dec. 2011, available at arXiv:1109.5484.

[9] B. Devillers and D. Gunduz, "A general framework for the optimization of energy harvesting communication systems with battery imperfections," Arxiv preprint arXiv:1109.5490, 2011.

[10] K. Tutuncuoglu and A. Yener, "Optimal power policy for energy harvesting transmitters with inefficient energy storage," to appear in Proceedings of 46th Annual Conference on Information Science and Systems, CISS, Princeton, NJ, Mar 2012.

[11] V. Sharma, U. Mukherji, V. Joseph, and S. Gupta, "Optimal energy management policies for energy harvesting sensor nodes," IEEE Transactions on Wireless Communications, vol. 9, no. 4, pp. 1326-1336, 2010.

[12] J. Lei, R. Yates, and L. Greenstein, "A generic model for optimizing single-hop transmission policy of replenishable sensors," IEEE Transactions on Wireless Communications, vol. 8, no. 2, pp. 547-551, 2009.

[13] P. Grover, K. Woyach, and A. Sahai, "Towards a communicationtheoretic understanding of system-level power consumption," IEEE Journal on Selected Areas in Communications, vol. 29, no. 8, pp. 1744$1755,2011$.

[14] A. Goldsmith and S. Chua, "Variable-rate variable-power mqam for fading channels," IEEE Transactions on Communications, vol. 45, no. 10, pp. 1218-1230, 1997.

[15] X. Qiu and K. Chawla, "On the performance of adaptive modulation in cellular systems," IEEE Transactions on Communications, vol. 47, no. 6, pp. 884-895, 1999. 